\title{
Strength of Coupling within a Mnemonic Control Network Differentiates Those Who Can and Cannot Suppress Memory Retrieval
}

\author{
Pedro M. Paz-Alonso, ${ }^{1,2,3 \star}$ Silvia A. Bunge, ${ }^{1,4 *}$ Michael C. Anderson, ${ }^{5,6}$ and Simona Ghetti ${ }^{2}$ \\ ${ }^{1}$ Helen Wills Neuroscience Institute, University of California, Berkeley, California 94720, ${ }^{2}$ Center for Mind and Brain, University of California, Davis, \\ California 95616, ${ }^{3}$ Basque Center on Cognition, Brain and Language, Donostia-San Sebastián, Gipuzkoa 20009, Spain, ${ }^{4}$ Department of Psychology, \\ University of California, Berkeley, California 94720, ${ }^{5} \mathrm{MRC}$ Cognition and Brain Sciences Unit, Cambridge CB2 7EF, United Kingdom, and ${ }^{6}$ Behavioural and \\ Clinical Neurosciences Institute, University of Cambridge, Cambridge CB2 3EB, United Kingdom
}

The ability to direct our thought processes influences not only what we do, but also what we remember later. Here we sought to identify the brain network that supports the ability to control memory retrieval and to understand the neural basis of age-related changes and individual differences in the capacity for mnemonic control. To this end, we collected functional MRI data from 43 children and young adults while they attempted to retrieve or suppress retrieval of previously learned associations. Seed-based functional connectivity analyses revealed a largely right-lateralized dorsolateral prefrontal cortex-cingulate-parietal-hippocampal network that exhibited strongly correlated activity during retrieval suppression. Regardless of age, individuals who were able to suppress memory retrieval exhibited tighter coupling between key nodes in this dorsolateral prefrontal cortex-cingulate-parietal-hippocampal network than individuals who did not. Further, only those capable of mnemonic control exhibited tighter coupling during successful retrieval suppression (intentional forgetting) than during unsuccessful retrieval (unintentional forgetting). Across both children and adults, individual differences in retrieval suppression were best explained by the strength of these network interactions.

\section{Introduction}

Dwelling on irrelevant memories is unproductive at best and counterproductive at worst. Thus, an important component of goal-directed behavior is the ability to focus our attention on memories that are relevant to our current goals. It has long been theorized that the ability to regulate memory retrieval has important benefits, not only for cognitive functioning but also for psychological well-being (Freud, 1966; Luria, 1968; Harnishfeger and Pope, 1996). Despite an active debate as to whether specific memories can be suppressed (Anderson and Green, 2001; Bulevich et al., 2006; Wegner, 2009; Depue, 2012; Detre et al. in press), there is

Received July 19, 2012; revised Jan. 9, 2013; accepted Jan. 29, 2013.

Author contributions: P.M.P.-A., S.A.B., M.C.A., and S.G. designed research; P.M.P.-A. performed research; P.M.P.-A. and S.A.B. analyzed data; P.M.P.-A., S.A.B., and S.G. wrote the paper.

This work was supported by a Juan de la Cierva Grant and Grant PSI2012-32093 from the Spanish Ministry of Economy and Competitiveness, and Grant PI2012-15 from the Department of Education, University and Research from the Basque Government (P.M.P.-A.), the National Science Foundation (Grant NSF 0448844 to S.A.B. and Grant NSF 0648564 to S.G.), and the James S. McDonnell Foundation (Scholar Awards to S.G. in 2010 and S.A.B. in 2011) We thank Natalie Repin and Megan Neal for assistance with data collection; Michael Souza and Carter Wendelken for technical assistance; Yuko Munakata and Art Shimamura for helpful discussions; Yee Lee Shing and Yana Fandakova for helpful comments on an earlier version of this manuscript; and the members of the Max Planck Institute for Human Development in Berlin for their support during the preparation of this manuscript.

The authors declare no competing financial interests.

*P.M.P.-A. and S.A.B. contributed equally to this work as joint first authors.

This article is freely available online through the J Neurosci Open Choice option.

Correspondence should be addressed to either Dr. Pedro M. Paz-Alonso or Prof. Silvia A. Bunge, 134 Barker Hall,

Helen Wills Neuroscience Institute, University of California, Berkeley, CA, 94720, E-mail: p.pazalonso@bcbl.eu or sbunge@berkeley.edu.

DOI:10.1523/JNEUROSCI.3459-12.2013

Copyright $\odot 2013$ the authors $\quad 0270-6474 / 13 / 335017-10 \$ 15.00 / 0$ mounting evidence that individuals can exert some measure of control over the process of memory retrieval (i.e., mnemonic control) remembering more of the items or associations that they are asked to remember than those that they are asked to forget (e.g., Wilson and Kipp, 1998; MacLeod, 1999; Depue et al., 2006; Hanslmayr et al., 2009; Joormann et al., 2009). As is the case for other forms of cognitive control, the capacity for mnemonic control waxes and wanes over the life span (Harnishfeger and Pope, 1996; Paz-Alonso et al., 2009; Anderson et al., 2011; Wendelken et al., 2011; but see Murray et al., 2011) and is subject to large individual differences (Levy and Anderson, 2008).

Brain imaging studies involving young adults show that attempts to stop memory retrieval are associated with greater activation of lateral prefrontal cortex (lPFC) than attempts to retrieve memories (Anderson et al., 2004; Depue et al., 2007, 2010; Butler and James, 2010; Benoit and Anderson, 2012; Levy and Anderson, 2012; for review, see Anderson and Huddleston, 2011), suggesting that control over the process of memory retrieval is an active, energy-consuming process, unlike the passive process of decay associated with forgetting. On the flipside, these studies show that attempts to suppress memory retrieval are associated with decreased activation in the hippocampus than attempts to retrieve memories, suggesting that hippocampal activity is downregulated during retrieval suppression.

Together, these prior results provide some clues regarding the neural basis of mnemonic control. However, they leave many questions unanswered. What, specifically, changes in a child's brain to support the emergence of mnemonic control? Why are 
some adults able to exert control over memory retrieval whereas others are not? And by what neural pathways does IPFC modulate activity in the hippocampus during mnemonic control? The present fMRI study of age-related changes and individual differences in mnemonic control provides novel insights and testable hypotheses with respect to these important questions.

Based on prior findings showing age-related differences in retrieval suppression (Paz-Alonso et al., 2009), we predicted that 8 - and 9-year-olds would fail to engage lPFC when asked to suppress retrieval, whereas 11 - and 12-year-olds would exhibit similar IPFC activation to adults. Contrary to this prediction, age-related differences were eclipsed by important individual differences within the age groups, and the capacity for mnemonic control was related not to IPFC activation levels but rather to the strength of temporal coupling among key nodes in a mnemonic control network.

\section{Materials and Methods}

\section{Overview of study design}

To study the neural basis of age-related changes and individual differences in mnemonic control, we adapted the original Think/No-Think (TNT) paradigm (Anderson and Green, 2001; Anderson et al., 2004), modifying the task design and word stimuli in the Bunge laboratory for use with children (Paz-Alonso et al., 2009). The TNT paradigm includes three phases: (1) an initial study phase, during which participants learn a set of word-word associations; (2) the TNT phase, during which they are shown one of the words and asked either to retrieve the associated word (Think trials) or to prevent the associated word from coming to mind (No-Think trials); and (3) the memory test phase, during which participants' memory for all the word pairs is assessed. Individuals who recalled fewer No-Think associations than Baseline associations when cued with the first word in a pair are referred to below as suppressors, and those who did not are referred to as nonsuppressors.

\section{Participants}

Participants were recruited from northern California (Davis, Sacramento, Berkeley, and San Francisco) through the Ghetti and Bunge laboratories at University of California Davis and University of California Berkeley. They received either monetary compensation or course credit for their participation. Before taking part in the experiment, all participants gave informed consent based on procedures approved by the Internal Review Board of the University of California Davis and Berkeley campuses. Children were prescreened with the Child Behavior Checklist (Achenbach, 1991).

The final study sample consisted of 43 right-handed native Englishspeaking participants from three age groups: 15 8- and 9-year-olds (mean, 9.1 years; range, 8.0-9.8 years; 8 females, 7 males), 1411 - and 12-year-olds (mean, 12.1 years; range, 11.2-12.9 years; 7 females, 7 males), and 14 young adults (mean, 22.4 years; range, 18.3-30.8 years; 6 females, 8 males). Data from 10 additional participants were excluded from analysis: one adult and one child obtained scores of 2 SD below the mean on the Wechsler Abbreviated Scale of Intelligence, and 8 child participants (five 8 - and 9-year-olds and three 11- and 12-year-olds) had head motion parameters of $>6 \mathrm{~mm}$ (twice the in-plane voxel dimensions) for at least one functional scan.

In addition to completing both the behavioral and MRI portions of the TNT task procedure, these participants also completed several standard cognitive measures: the Wechsler Abbreviated Scale of Intelligence as a measure of IQ, and two verbal working memory measures from the Woodcock-Johnson III Tests of Cognitive Abilities: Number Reversed and Auditory Working Memory (Woodcock et al., 2001). Additionally, the adult participants completed a standard computerized Go/No-Go test with $80 \%$ Go stimuli, $20 \%$ No-Go stimuli, $600 \mathrm{~ms}$ presentation rate, so that we could test for a correlation between mnemonic and response control. As we report below, performance on the Go/No-Go task and the other cognitive assessments was unrelated to the capacity for mnemonic control.

\section{TNT stimuli}

The test materials used in the present study were previously used in a behavioral study with children aged 8 to 12 years of age and adults (PazAlonso et al., 2009). The study procedure was similar to that used in prior TNT studies with adults (e.g., Anderson et al., 2004), but we adapted the stimuli for use with children aged 7 years and older on the basis of published word acquisition norms (Coltheart, 1981). A total of 56 word pairs (e.g., Vacation-Palm) were included in the present study. The two words in each cue-target pair were designed to be weakly semantically related, with none of the association strength values $>0.025$ (Nelson et al., 2004). Each target word (i.e., right-hand pair member) belonged to a unique semantic category, and the association between targets and category cues was equated to that of the word pairs.

Twenty of these word pairs were used as examples and as practice trials, as detailed below. The remaining 36 pairs were divided into three stimulus sets of 12 word pairs each that were rotated through Think, No-Think, and Baseline conditions across participants. The first word of each of the 12 Think and 12 No-Think word pairs was presented 15 times during scanning, yielding fMRI data for 180 trials during which participants attempted to retrieve the associated word and another 180 trials during which they attempted to suppress retrieval of the associated word.

\section{Experimental procedure}

Study phase (outside the scanner). Participants first learned the 56 word pairs outside the scanner, their memory was tested up to three times, and the correct response was provided after each retrieval attempt. Word pairs were presented visually for $5 \mathrm{~s}$, and the study test feedback procedure was repeated up to three times as needed. By design, the percentage of correctly recalled target words on the final test during this study phase did not differ statistically across age groups $\left(F_{(2,40)}=1.26 ; p=0.29 ; 8\right.$ and 9-year-olds, $63 \pm 9 \%$, mean \pm SD; 11 - and 12-year-olds, $68 \pm 10 \%$; adults, $63 \pm 10 \%)$. Because participants were provided with the correct pairings again after being tested on them for the last time, these estimates of the number of word pairs learned during the study phase are in all likelihood overly conservative.

TNT phase. After the study phase, we collected fMRI data for the TNT phase of the experiment, during which participants were presented with the left-hand member of each Think and No-Think word pair, and were cued either to retrieve the associated memory or to prevent the associate from coming to mind. Participants received instructions on the TNT phase of the task and participated in a 7 min practice phase that resembled the actual TNT task that they would perform later in the scanner. They learned that, if a word was presented in green, they should attempt to retrieve its associate, but that if it was presented in red, they should try not to. During this practice phase, participants practiced retrieval and retrieval suppression with 8 Think and 8 No-Think trials, randomly presented. This practice phase was intended to ensure that both children and adults understood the task instructions and had an opportunity to practice implementing them before data collection.

After this practice phase, participants were moved into the MRI scanner, where they underwent 5 min of anatomical scans before performing three $8 \mathrm{~min}, 24 \mathrm{~s}$ fMRI runs of the TNT task, viewing individual words on the screen and attempting to retrieve or not retrieve the associated word. On each run, participants encountered five repetitions of 24 item blocks, including 12 Think trials and 12 No-Think trials, for a total of 15 repetitions per item (where each item was the cue word from a cue-target word pair). On each trial, a cue from one of the pairs appeared for $2.7 \mathrm{~s}$ in green or in red, followed by a $300 \mathrm{~ms}$ intertrial interval. When the cue was presented in green, participants were instructed to recall the second member of the word pair (Think trials). When the cue was presented in red, participants were instructed to keep the target word out of mind while still focusing on the cue word (No-Think trials). Think and NoThink stimuli were pseudorandomly intermixed within each block, with no more than four consecutive items from either category.

Memory test phase (outside the scanner). After the scan session, participants were asked to take two tests that assessed their memory for the right-hand member of all initially presented word pairs, as described below. Think, No-Think, and Baseline items were intermixed during this test phase, and there was no indication as to which condition a given 
stimulus pertained to (unlike the TNT phase, in which Think and NoThink items were printed in different colors). Critically, although participants had been instructed previously not to remember the right-hand members of the No-Think pairs, they now were now encouraged to do so and were told that they would receive 10 cents for each correct answer.

We sought to test whether participants' explicit memory for the learned word pairs was modulated by the task instructions. Thus, for each studied word pair, participants were given the previously studied cue word (e.g., Vacation) for $4 \mathrm{~s}$ and were asked to recall the target word aloud (e.g., Palm). After participants completed this "same-probe" memory task, they also completed an incidental memory test for the target word (Independent-Probe test), in which they were given a category cue and the first letter of the target word (e.g., Tree-P__). This "independent-probe task" has been used previously to test whether the memory for the right-hand members of the word pairs is more difficult to access after repeated attempts to block their retrieval (Anderson and Green, 2001). However, we have shown that children have more difficulty than adults when attempting to retrieve the target word with a probe that differs from that used during initial learning and during the TNT phase (Paz-Alonso et al., 2009). Thus, the same-probe memory test reported here is a more accurate measure of child's memory for the initially learned word pair (For more details, see the supplemental Materials of Paz-Alonso et al., 2009).

\section{fMRI data acquisition}

Whole-brain fMRI data acquisition was conducted on 3-T Siemens TRIO whole-body MRI scanners (Siemens Medical Solutions) at the University of California Davis Imaging Research Center and at the University of California San Francisco Neuroscience Imaging Center, using a standard whole-head coil. To limit head movement, the area between participants' heads and the head coil was padded with foam, and participants were asked to remain as still as possible. Snugly fitting headphones were used to dampen background scanner noise and to enable communication with experimenters while in the scanner. Participants viewed stimuli back-projected onto a projection screen with a mirror mounted on the head coil.

Functional images were acquired using a gradient-echo echo-planar pulse sequence $(\mathrm{TR}=3000 \mathrm{~ms}, \mathrm{TE}=25 \mathrm{~ms}, 34$ high in-plane resolution axial slices, $1.7 \times 1.7 \times 3.4,0 \mathrm{~mm}$ interslice gap, flip angle $=90^{\circ}$, field of view $=220 \mathrm{~mm}, 164$ volumes per run). The order of Think and NoThink trials and intertrial intervals (jitter fixation, 30\% of total trials) within each functional run were determined with an algorithm designed to maximize the efficiency of the recovery of the blood oxygen leveldependent response (Optseq II) (Dale, 1999). Before each scan, four volumes were discarded to allow for T1 equilibration effects. Coplanar T2-weighted and high-resolution T1-weighted anatomical images were also collected. The order of scans was as follows: T2 localizer scan, T2 coplanar scan, 3 functional echo-planar imaging scans, and a highresolution three-dimensional T1 MPRAGE scan.

\section{Preprocessing of $\mathrm{FMRI}$ data}

Standard SPM5 (Wellcome Department of Cognitive Neurology, London) preprocessing routines and analysis methods were used. The first four volumes of each functional scanning run were discarded to allow for T1 equilibration. Functional images were corrected for differences in slice acquisition timing and were realigned to the first volume by means by rigid-body motion transformation. Motion parameters were extracted from this process and were used to inform additional motion correction algorithms implemented by the Artifact Repair toolbox (ArtRepair; Stanford Psychiatric Neuroimaging Laboratory) intended to repair outlier volumes with sudden scan-to-scan motion exceeding 2 $\mathrm{mm}$ and volumes whose global intensity was $>2$ SD away from the mean (Mazaika et al., 2009). After volume repair, structural and functional volumes were spatially normalized to T1 and echo-planar imaging templates, respectively. The normalization algorithm used a 12-parameter affine transformation together with a nonlinear transformation involving cosine basis functions. During normalization, the volumes were resampled to 3-mm-cubic voxels. Templates were based on the MNI305 stereotaxic space (Cocosco et al., 1997), an approximation of Talairach space (Talairach and Tourneaux, 1988). These procedures have been validated for use in children 6 years of age and older (e.g., Burgund et al., 2002; Kang et al., 2003). After normalization, functional volumes were spatially smoothed with an $8 \mathrm{~mm}$ full width at half-maximum isotropic Gaussian kernel.

\section{fMRI data analysis}

Statistical analyses performed on individual participants' data using the general linear model (GLM). fMRI time series data were modeled by a series of impulses convolved with a canonical hemodynamic response function. The motion parameters for translation (i.e., $x, y, z)$ and rotation (i.e., yaw, pitch, roll) were included as covariates of noninterest in the GLM. Each trial was modeled as an event, time-locked to the onset of the cue period. The resulting functions were used as covariates in a GLM, along with a basic set of cosine functions that high-pass filtered the data, and a covariate for session and scanner effects. The least-squares parameter estimates of the height of the best-fitting canonical hemodynamic response function for each condition were used in pairwise contrasts. Contrast images, computed on a participant-by-participant basis, were submitted to group analyses.

At the group level, whole-brain contrasts between conditions were computed by performing one-tailed $t$ tests on these images, treating participants as a random effect. Our standard statistical threshold was a false discovery rate $(F D R)$ set to $\mathrm{q}<0.05$. On several occasions, we note that no voxels met the standard threshold but present the results obtained when the statistical threshold is relaxed to $p<0.001$ uncorrected with cluster-level correction for multiple comparisons at $p<0.01$. These uncorrected analyses have yielded potential insights that should be confirmed in a subsequent study. Region of interest (ROI) analyses were performed with the MARSBAR toolbox for use with SPM5. Throughout the manuscript, brain coordinates correspond to the MNI coordinates for the center of mass of a cluster.

\section{Functional connectivity analyses with left and right hippocampal} seed regions

To better understand the neural circuitry supporting hippocampal modulation during mnemonic control, we conducted whole-brain $\beta$-series functional connectivity analyses across all participants for the All trials $>$ Null contrast, and tested for differential coupling between the four main study conditions (Think-remembered, Think-forgotten, No-Thinkremembered, and No-Think-forgotten), age groups (8- and 9-year-olds, 11 - and 12-year-olds, and young adults), and performance groups (suppressors and nonsuppressors).

We measured the strength of temporal coupling between brain regions via the $\beta$-series correlation method for fMRI data (Rissman et al., 2004), implemented in SPM5 with custom Matlab scripts. The canonical hemodynamic response function in SPM was fit to each occurrence of each condition, and the resulting parameter estimates (i.e., $\beta$ values) were sorted according to the study conditions to produce a condition-specific $\beta$-series for each voxel. Left and right hippocampal ROIs identified from the All $>$ Null contrast were used as separate seed regions to identify clusters throughout the brain that exhibited temporally coupled fluctuations in activation with left or right hippocampus ( $p<1 \mathrm{e}-9)$. Next, in accordance with previous neuroimaging evidence suggesting that effective mnemonic control requires modulation of bilateral hippocampus, we conducted a conjunction analysis to specifically characterize the clusters that were tightly correlated with both the left and right hippocampal seed regions. Although a similar set of clusters was obtained when this connectivity analysis was restricted to No-Think $>$ Null trials, we sought to eliminate bias in cluster selection: identifying a general network based on patterns of connectivity across both Think and No-Think trials made it possible to test for differences in functional connectivity between these task conditions.

\section{Results}

\section{Behavioral results}

Because the focus of this study was on brain activation measured while participants attempted memory retrieval or retrieval suppression, we report first on the two conditions for which we 
acquired MRI data: Think and No-Think. We conducted a 3 (age group: 8- and 9-year olds, 11- and 12-year olds, adults) $\times 2$ (condition: No-Think, Think) mixed-model ANOVA with percentage recalled in the Same-Probe memory test as the dependent measure. This analysis revealed main effects of age group $\left(F_{(2,40)}=\right.$ 5.12; $p<0.05)$ and condition $\left(F_{(1,40)}=\right.$ 26.23; $p<0.001)$, with an Age group $\times$ Condition interaction that approached significance $\left(F_{(2,40)}=2.82 ; p=0.07\right.$; significant one-tailed for the expected direction of age-related differences). Consistent with the results of our larger behavioral study $(N=70)$ (Paz-Alonso et al., 2009; Fig. 1A), we observed a trend toward an age-related improvement in mnemonic control in this sample $(N=$ 43; Fig. $1 B$ ). Nevertheless, the large variability within age groups warranted closer attention.

In addition to subdividing participants as a function of age, we also subdivided them as a function of their capacity for mnemonic control. Prior behavioral work involving the TNT paradigm in adults (Levy and Anderson, 2008; Anderson and Huddleston, 2011) revealed greater individual differences among adults for retrieval suppression (recall for Baseline vs No-Think associations) than for retrieval enhancement (recall for Think vs Baseline associations).

Thus, we defined as suppressors those individuals for whom the recall ratio of (Baseline-No-Think)/Baseline) word pairs on the Same-Probe memory test was positive $(N=19$ across age groups; 9 female, 10 male). By contrast, we defined as nonsuppressors those with zero or negative recall ratios on this same memory test $(N=24$ across age groups; 12 female, 12 male). On the basis of this performance criterion, only $33 \%$ of the 8 - and 9 -year-olds in our sample were categorized as suppressors, compared with $50 \%$ of the 11 - and 12 -year-olds and $50 \%$ of the adults. Figure $1 C$ shows percentage recalled and its distribution as a function of memory suppression group and study conditions.

To verify that the two performance groups did indeed exhibit significant differences in retrieval suppression, as intended, we conducted a 2 (Performance group: suppressors vs nonsuppressors) $\times 2$ (Condition: No-Think, Baseline) mixed-model ANOVA with percentage recalled in the Same-Probe memory test as the dependent measure. This analysis confirmed that the main effect of condition $\left(F_{(1,41)}=18.70 ; p<0.001\right)$ was qualified by a significant Group $\times$ Condition interaction $\left(F_{(1,41)}=86.56\right.$; $p<0.001$ ), indicating that suppressors did indeed exhibit a significantly lower percentage of recall for No-Think than Baseline items relative to nonsuppressors, who did not this not show differences between these condition levels.

Notably, suppressors and nonsuppressors did not differ in age $\left(t_{(1,41)}=0.26, p=0.45\right)$, IQ as measured by the Wechsler Abbreviated Scale of Intelligence $\left(t_{(1,40)}=1.12, p=0.27\right)$, or gender $\left(\chi_{(1,29)}^{2}=0.03, p=0.86\right)$. Nor did they differ on verbal working memory, as measured by Auditory Working Memory and Numbers Reversed $\left(F_{(1,41)}=1.76 ; p=0.19\right)$. Within each age group, there were also no statistically significant differences between
A Prior behavioral study $(\mathrm{N}=70)$

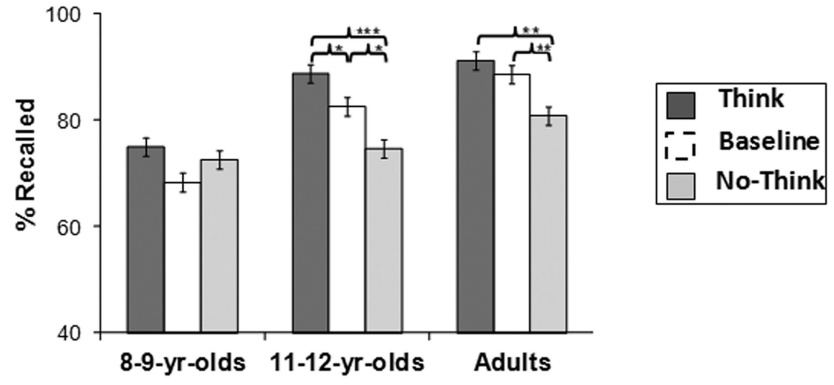

Individual differences $(\mathrm{N}=43)$

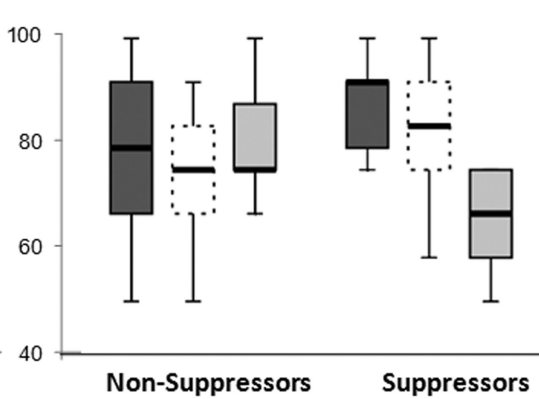

Figure 1. Behavioral evidence of mnemonic control during the TNT phase as measured by subsequent memory recall (i.e.,

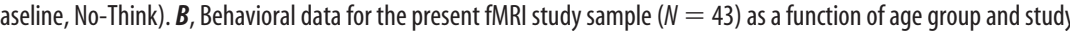
ressors) and study condition. $\boldsymbol{B}, \boldsymbol{C}$, Dotted outlines indicate the condition (Baseline) for which fMRI data were not

suppressors and nonsuppressors in terms of IQ, working memory, or gender $(p>0.05)$. Among adults, who unlike the children, additionally completed a Go/No-Go test of response control, we found that suppressors and nonsuppressors did not differ in terms of errors of commission on No-Go trials $\left(F_{(1,11)}=\right.$ $0.69 ; p=0.43)$, or response times on correctly performed Go trials $\left(F_{(1,11)}=0.03 ; p=0.86\right)$.

Thus, there were important individual differences in mnemonic control across our sample that could not be explained by differences in IQ, working memory, or, in the case of adults, response control. To further explore these individual differences, we conducted fMRI analyses comparing suppressors and nonsuppressors (collapsing across age groups) as well as analyses comparing age groups.

\section{fMRI results}

Whole-brain contrasts for No-Think versus Think trials across participants

First, to measure overall differences in activation for attempted retrieval and retrieval suppression, we computed whole-brain fMRI contrasts for Think versus No-Think trials across all participants (Fig. $2 A$; FDR corrected at $\mathrm{q}<0.05$ ). We observed reduced activation on No-Think relative to Think trials in bilateral hippocampus, as well as in bilateral ventral precuneus (BA 7) and posterior cingulate cortex (BA 23/31). On the other hand, we observed increased activation on NoThink relative to Think trials in a largely right-lateralized pattern of brain activation that included clusters in IPFC (BA 44/45/9/10), basal ganglia (right globus pallidus, bilateral putamen), dorsal precuneus (BA 7), and left and right lateral posterior parietal cortex (1PPC, BA 40). 


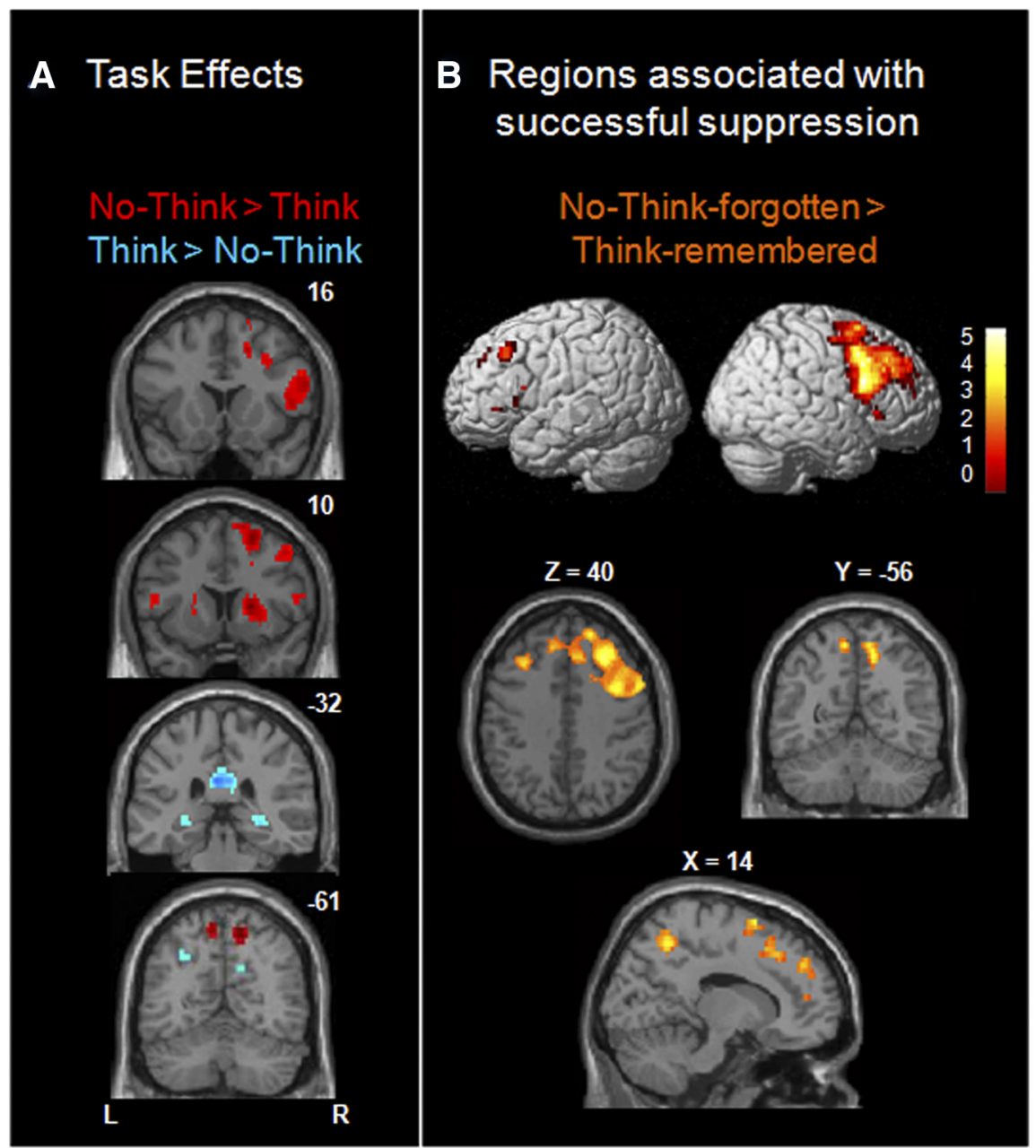

Figure 2. Whole-brain contrasts comparing attempts with retrieve versus suppress retrieval, across all participants. $\boldsymbol{A}$, Task effects: No-Think $>$ Think (red) and the opposite Think $>$ No-Think (blue) contrasts in slice sections along the $y$-axis. $\boldsymbol{B}$, Regions associated with successful suppression. Brain renderings and slice sections showing activation for successful retrieval suppression relative to successful retrieval (i.e., No-Think-forgotten $>$ Think-remembered trials).

These findings, based on our entire sample of children and adults, are broadly consistent with prior adult fMRI studies in showing IPFC engagement during No-Think versus Think trials with a concomitant reduction in medial temporal lobe (MTL) activation (Anderson et al., 2004; Depue et al., 2007; Benoit and Anderson, 2012; Levy and Anderson, 2012). We note additionally that different regions within parietal cortex were engaged for Think $>$ No-Think items (bilateral ventral precuneus, BA 7) and for No-Think $>$ Think items (bilateral dorsal precuneus, BA 7) and IPPC, BA40; Figure 2A). As shown below, IPPC appears to play a central role in mnemonic control.

Whole-brain contrasts for No-Think-forgotten versus Thinkremembered trials across participants

The contrast between Think and No-Think trials does not take into account the fact that not all attempts to retrieve or to suppress memories are successful and that individual participants differ in their capacity for mnemonic control. Thus, we sought to classify fMRI trials not only as a function of task instructions (Think, No-Think), but also as a function of individual participants' subsequent memory (remembered, forgotten) for word pairs on the Same-Probe memory test conducted immediately after the scan session. A direct comparison between successfully forgotten (No-Think-forgotten) and successfully remembered (Think- remembered) trials is shown in Figure $2 B$. Despite the reduced number of trials included in this more selective analysis, the right $\mathrm{IPFC}$ activation was even more robust than in Figure $2 A$, and we observed additional suprathreshold voxels in dorsolateral PFC (dlPFC; BA 9 and BA 46): 267 voxels, compared with 59 voxels for the more general contrast of No-Think $>$ Think trials. When we computed this contrast separately for each age group using cluster-level correction (see Materials and Methods), we found that all three age groups engaged right-hemisphere-dominant IPFC for NoThink-forgotten $>$ Think-remembered trials.

\section{Analyses focused on the MTLs across participants}

After conducting these whole-brain contrasts, we sought to look more closely within the MTL to measure modulation by task instructions (Think, No-Think) and subsequent memory (Think-remembered, Think-forgotten). As such, we conducted several contrasts limiting our search space to the MTL using an anatomical mask, FDR corrected at $\mathrm{q}<0.05$ (Fig. 3). Both the Think $>$ No-Think and Think-remembered $>$ No-Think-forgotten masked contrasts revealed several clusters in bilateral hippocampus and parahippocampal gyrus across the three age groups, confirming that several regions in the MTL were modulated by the task instructions. As shown in Figure 3 , hippocampal voxels that were most strongly modulated by task were, generally speaking, located posterior relative to voxels that were modulated most strongly by subsequent memory (Remembered $>$ Forgotten, collapsing across Think and No-Think trials). Although these voxelwise analyses were restricted to an anatomical mask of the MTL, the hippocampal and parahippocampal clusters shown in Figure 3 were also significant when correcting for multiple comparisons at the whole-brain level (FDR-corrected, $\mathrm{q}>0.05$ ) and at the cluster level $(p<0.01)$.

\section{Testing for differences in activation between groups defined} by age or performance

Having identified the regions that were modulated by task demands (TNT and Think-remembered/No-Think-forgotten) across participants, we sought to test for differences in activation between age groups and performance groups. However, neither whole-brain nor MTL-restricted two-sample $t$ tests comparing pairs of age groups or performance groups yielded significant differences at FDR, $p<0.05$.

Using cluster-level correction, an age-related difference in activation on No-Think-forgotten $>$ Think-remembered trials was observed in right IPPC. A direct whole-brain comparison between the most distant age groups revealed stronger No-Think-forgotten $>$ Think-remembered activation for adults than 8- and 9-year-olds in this region (40 voxels in BA 40, peak at 38/-48/60; 39 voxels in BA 7, peak at 28/-54/60). This age-related difference held when perfor- 
mance differences in mnemonic control between groups were partialled out. Right IPPC was also the only region to exhibit a cluster-corrected positive correlation with age (18 voxels in BA 40, peak at $38 /-50 / 60 ; 49$ voxels in BA 7 , peak at 26/-58/60). Again, this age effect in PPC held when differences in performance were partialled out.

Overall, we observed only minimal differences in activation between age groups. Notably, the region that best differentiated the 8- and 9-year-olds' and adults' brain activation for successfully forgotten versus successfully remembered associations was located in IPPC, and not in dlPFC, as we had predicted on the basis of prior fMRI studies highlighting the role of this latter region in retrieval suppression.

These univariate group comparisons, followed by ROI analyses testing for group differences in level of activation, led us conclude that what distinguishes those who can and cannot regulate memory retrieval is not fully captured, at least in the present mixed sample of children and adults, by differences in level of blood oxygen level-dependent activation. Next, we sought to test for differences in network connectivity between age groups and between performance groups. In particular, we predicted that suppressors of all ages would exhibit tighter connectivity between IPFC and the MTL than nonsuppressors. In preparing to test this hypothesis, we first needed to characterize the functional network involving MTL and PPFC that supports retrieval suppression.

\section{Identifying a mnemonic control network across participants}

To identify clusters exhibiting tight temporal coupling with both left and right hippocampal seeds across trials, we conducted wholebrain $\beta$-series seed-based functional connectivity analyses. We used as seeds left and right hippocampal ROIs derived from the contrast of All trials $>$ Null across all participants to identify regions exhibiting a correlation with these regions at $p<1 \mathrm{e}-9$. A similar set of clusters was obtained when the connectivity analysis was restricted to No-Think trials. However, we elected to identify a general network based on patterns of connectivity across all trials because it allowed us to directly compare in an unbiased manner the strength of network connectivity for the 4 main study conditions: Thinkremembered, Think-forgotten, No-Think-remembered, and NoThink-forgotten. As shown in Figure $4 A$, clusters exhibiting tight coupling with bilateral hippocampus were identified in middle occipital cortex, precuneus (BA 7), superior parietal cortex (BA 7), middle temporal cortex, parahippocampal gyri, fusiform gyri, posterior and mid-cingulate cortex (BA 31/24), thalamus, superior frontal cortex (BA 8), and dlPFC (BA 9).

\section{Testing for age and performance differences in average} network strength

Seeking to test for task differences and group differences in overall strength of connectivity across the mnemonic control net- work, we built 5-mm-radius spheres centered in the 61 local maxima identified as being functionally coupled with bilateral hippocampus for all trials $>$ null (Fig. $4 A$ ). For each participant, we extracted the correlation strength ( $r$ value) for each pair of regions and each of the main study conditions. We calculated an average network connectivity value for each participant from the pairwise correlations among the 61 spherical ROIs and per each study condition. These average network connectivity values were normally distributed, according to the Shapiro-Wilk test of normality (all, $p>0.05$ ). Using these values, we conducted mixed ANOVAs to test for differences in overall network strength between age groups and performance groups.

First, we tested for significant age-related differences in average network connectivity. A 3 (age group: 8- and 9-year-olds, 11 - and 12-year-olds, young adults) $\times 2$ (Task: Think, No-Think) $\times$ 2 (Memory: remembered, forgotten) mixed ANOVA for average network strength revealed main effects of Task $\left(F_{(1,39)}=4.38\right.$; $p<0.05)$, and Memory $\left(F_{(1,39)}=24.38 ; p<0.001\right)$ that were qualified by a significant Age group $\times$ Memory interaction $\left(F_{(2,39)}=6.21 ; p<0.01\right)$. Overall, coupling strength across subjects was higher for No-Think $(0.28 \pm 0.01$, mean \pm SD) than for Think trials $(0.26 \pm 0.01)$, as well as for remembered $(0.28 \pm$ $0.01)$ relative to forgotten trials $(0.26 \pm 0.01)$. Post hoc analyses 

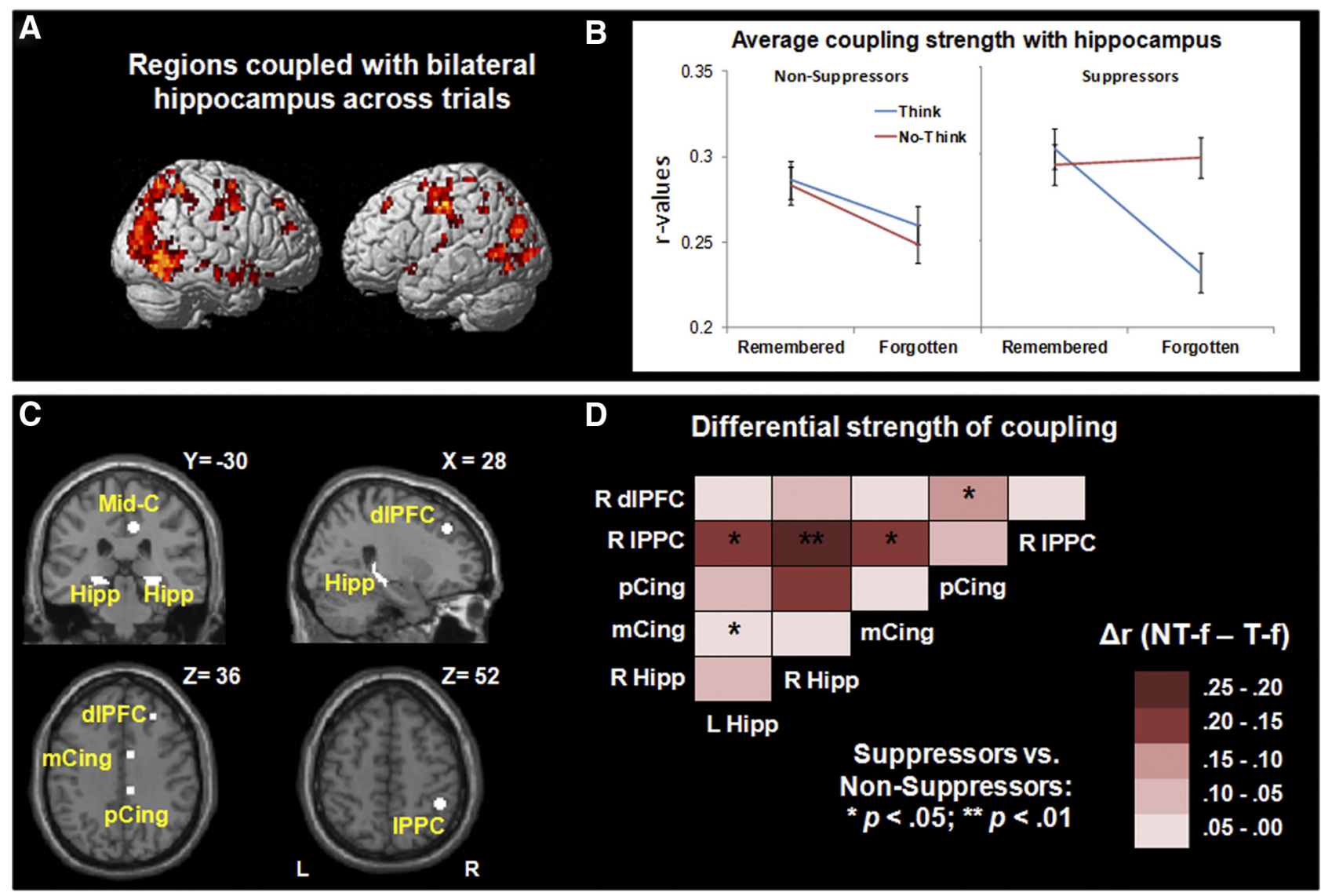

Figure 4. Functional connectivity analyses for all trials relative to Baseline. $A$, Brain renderings showing clusters identified from the conjunction analysis of the clusters tightly correlated, across the entire brain and participants, with both left and right hippocampus seeds for All $>$ Null. B, Average coupling strength between all the clusters tightly correlated with hippocampus as a function of Suppression group, Task, and Memory. Average coupling strength was higher for No-Think-forgotten than for Think-forgotten conditions, only for those participants who did exhibit behavioral memory suppression effects. C, ROls tightly correlated with hippocampus used in subsequent functional connectivity pairwise correlations, including left and right hippocampus (Hipp), middle cingulate (mCing), posterior cingulate (pCing), right lateral posterior parietal cortex (IPPC), and right dIPFC seeds. D, Correlation matrices showing differences in pairwise correlation strength between pairs of ROls described in C for No-Think-forgotten-Think-forgotten for suppressors versus nonsuppressors.

revealed that the interaction between Age group and Memory factors was because, in children, overall strength of network coupling was similar for successfully remembered (i.e., Thinkremembered, $0.29 \pm 0.01$ ) and unsuccessfully remembered (NoThink-remembered, $0.29 \pm 0.01 ; p=0.86)$ items and therefore exhibited a significant remembered $(0.29 \pm 0.01)>$ forgotten effect $(0.25 \pm 0.02 ; p<0.001)$ across Think and No-Think conditions that was not observed for adults (remembered, $0.27 \pm$ 0.02 ; forgotten, $0.26 \pm 0.02 ; p=0.41$ ). This age difference remained statistically significant when the number of trials per condition was entered as a covariate in the analysis.

Next, we tested for significant performance-related differences in average network connectivity. A 2 (Performance group: suppressors, nonsuppressors) $\times 2$ (Task: Think, No-Think) $\times 2$ (Memory: remembered, forgotten) mixed ANOVA that included participants' age as a covariate revealed a main effect of Memory $\left(F_{(1,39)}=4.97 ; p<0.05\right)$, a significant Performance group $\times$ Task interaction $\left(F_{(1,39)}=7.09 ; p<0.05\right)$, and a significant Performance group $\times$ Task $\times$ Memory interaction $\left(F_{(1,39)}=6.14\right.$; $p<0.05$ ) (Fig. 4B). Overall, coupling strength was higher for remembered than for forgotten trials across participants. Post hoc analysis revealed that the three-way interaction was driven by stronger coupling for the No-Think-forgotten condition relative to the Think-forgotten condition among suppressors $(p<0.01)$ but not among nonsuppressors $(p=0.55)$. This difference between performance groups remained statistically significant when the number of trials per condition was entered as a covariate in the analysis. Thus, only suppressors exhibited stronger average coupling within the mnemonic network for intentional than unintentional forgetting (NoThink-forgotten vs Think-forgotten).

Importantly, when this functional connectivity analysis in overall strength of connectivity was conducted with the set of clusters identified as tightly coupled with bilateral hippocampus for NT trials $>$ null, similar effects were found for the ANOVA testing for differences in coupling between age groups (i.e., main effect of Memory, $F_{(1,39)}=26.81 ; p<0.001$, and a Age group $\times$ Memory interaction, $\left.F_{(2,39)}=9.06 ; p<0.01\right)$, and between performance groups (i.e., main effect of Memory, $F_{(1,39)}=9.21 ; p<0.01$, a Performance group $\times$ Task interaction, $F_{(1,39)}=6.96 ; p<0.05$, and a Performance group $\times$ Task $\times$ Memory interaction, $F_{(1,39)}=$ 5.21; $p<0.05)$. Post hoc analysis for the higher order interactions yielded identical effects to the ones reported for overall strength of connectivity when clusters tightly coupled with bilateral hippocampus were identified for All trials $>$ null.

Testing for performance differences in strength of connectivity between key network nodes

The analyses above revealed age- and performance-related differences in overall strength of coupling within the mnemonic con- 
trol network. In particular, the performance-based ANOVA revealed a Group $\times$ Task $\times$ Memory interaction that survived when controlling for age and for number of trials. Next, we sought to explore this three-way interaction more carefully by measuring the strength of coupling between key nodes in the network for suppressors and nonsuppressors.

To this end, we selected six ROIs from the pool of regions that were tightly coupled with bilateral hippocampus that were observed in relevant whole-brain contrasts in the present study (i.e., All $>$ Null, Think $>$ No-Think, No-Think $>$ Think, No-Thinkforgotten $>$ Think remembered) and that were also close to local maxima reported in previous TNT neuroimaging studies (Anderson et al., 2004; Depue et al., 2007; Butler and James, 2010). These ROIs were located in left and right hippocampus and rightlateralized mid-cingulate (BA 24), posterior cingulate (BA 31), IPPC (BA 40), and dlPFC (BA 9) (Fig. 4A,C).

We focused on differential pairwise correlations between these nodes for No-Think-forgotten versus Think-forgotten trials, as these were the conditions that had driven the Performance group $\times$ Task $\times$ Memory interaction observed for average functional connectivity (Fig. 4B). Building on the previous average network strength analysis, pairwise comparisons allowed us to examine the specific role of distributed key nodes along the network and their interactions, as well as to determine the extent to which differential coupling strength among them qualifies the results observed in the prior average network analysis. We extracted the average differential correlation strength for NoThink-forgotten minus Think-forgotten conditions for each pair of ROIs for each participant, and transformed them in Fisher- $Z$ normally distributed values. Then, we conducted two-sample $Z$ tests on these values to test for significant differences in correlation strength between performance groups.

Indeed, as shown in Figure 4D, suppressors exhibited stronger differential pairwise connectivity than nonsuppressors for intentional versus nonintentional forgetting in key nodes of the network. For all of the statistically significant group differences (FDR corrected at $\mathrm{q}<0.05$ for multiple comparisons), suppressors exhibited stronger differential coupling than nonsuppressors, specifically for the following pairs of regions: bilateral hippocampus-right IPPC; left hippocampus-mid-cingulate; mid-cingulate-lPPC; and posterior cingulate-dlPFC. Thus, suppressors of all ages exhibit tighter coupling than nonsuppressors among the hippocampus, posterior and mid-cingulate, IPPC, and dIPFC during successful retrieval suppression.

In summary, the key findings reported above are as follows. First, our behavioral data revealed a developmental trend toward stronger retrieval suppression. However, strong individual differences in retrieval suppression were present in all three age groups. Whole-brain contrasts across all participants replicated prior findings for adults, in that hippocampus and parahippocampal gyrus were less active on No-Think than Think trials, whereas IPFC exhibited the opposite pattern. Focusing more specifically on successfully performed trials, we noted that all three age groups exhibited right IPFC activation during successful retrieval suppression relative to successful retrieval. Examining the MTL activations more closely, we found that the hippocampus was, across participants, modulated both by task demands and subsequent memory. Group comparisons involving the three age groups or the two performance groups (suppressors and nonsuppressors) revealed minimal differences in whole-brain contrasts, with the exception of an age-related increase in right IPPC activation for successful retrieval suppression versus successful retrieval. Turning to functional connectivity analyses, we found that IPFC, cingulate, and IPPC regions were tightly coupled with the hippocampus across trials. Suppressors, but not nonsuppressors, exhibited stronger average network coupling for intentional than unintentional forgetting (No-Think-forgotten $>$ Thinkforgotten). Further, suppressors exhibited stronger pairwise correlations than nonsuppressors for intentional versus unintentional forgetting among key nodes within the prefrontal-cingulateparietal-hippocampal network.

\section{Discussion}

The initial goal of this study was to examine the neural substrates underlying development of control over memory retrieval. Based on our prior behavioral research showing age-related differences in retrieval suppression (Paz-Alonso et al., 2009), we predicted that 8- and 9-year-olds would fail to engage 1PFC when asked to suppress retrieval, whereas 11- and 12-year-olds would exhibit similar IPFC activation to adults. Indeed, however, our analyses (both behavioral and neural) reveal a more nuanced account of retrieval suppression than we had initially envisioned.

Behaviorally, we observed a trend toward age-related improvements in retrieval suppression, based on the fact that only one-third of 8- and 9-year-olds exhibited retrieval suppression, compared with one-half of 11- and 12-year-olds and one-half of adults. However, it is necessary to point out the strong individual differences within each of the three age groups: in particular, the fact that only $50 \%$ of adults demonstrated effective retrieval suppression on this task, consistent with prior reports of large individual variability in mnemonic control (Levy and Anderson, 2008).

Indeed, we discovered that individual variation in retrieval suppression was a more important factor than age per se in this pool of mixed-age participants. Participants categorized as suppressors versus nonsuppressors did not differ in age, and the magnitude of the behavioral suppression effect for suppressors did not differ across the three age groups. Thus, large individual differences in mnemonic control, reported previously for adults (Levy and Anderson, 2008), are observed already by age 8 . We have shown previously, in a behavioral study involving 40 children $8-12$ years of age and 30 young adults, that retrieval suppression is positively correlated with age from 8 to 12 years, consistent with a large body of literature showing improved cognitive control over this age range (Luna et al., 2004; Davidson et al., 2006: Bunge and Wright, 2007). Thus, either a cross-sectional fMRI study with a larger sample or, better yet, a longitudinal study examining within-person changes over middle childhood would likely reveal stronger age-related differences than we have observed here.

Neurally, we replicated prior studies (Anderson et al., 2004; Depue et al., 2006) by showing that, across participants, a large swath of IPFC was more active on No-Think than Think trials, and also for No-Think-forgotten vs Think-remembered trials, whereas the hippocampus was more active for the reverse contrasts.

We found that age-related and individual differences in retrieval suppression were best explained not by differences in level of activation but rather by strength of functional connectivity within a frontal-cingulo-parietal-hippocampal network. Further, regardless of age, individuals whose attempts at retrieval suppression proved effective exhibited stronger coupling within the mnemonic control network on No-Think trials than individuals whose attempts were unsuccessful.

Prior work indicates that various parietal subregions support retrieval from long-term memory (e.g., Cabeza et al., 2008; 
Hutchinson et al., 2009; Olson and Berryhill, 2009; Shimamura, 2011). The current findings indicate that parietal cortex also plays a key role in the suppression of memory retrieval. Our functional connectivity results suggest that parietal cortex is a key intermediary in the modulation of the hippocampus by IPFC during retrieval suppression, as during retrieval (Shimamura, 2011). Further, whole-brain contrasts across participants revealed that IPPC and dorsal precuneus were more strongly engaged on NoThink than Think trials, whereas ventral precuneus showed the opposite pattern. Finally, the most significant difference in activation between adults and 8 - and 9-year-olds was located in right IPPC (BA 7, 40). Together, these three analyses support the hypothesis that mnemonic control arises from local interactions within parietal cortex between neurons conveying top-down (frontal, parietal) and bottom-up (MTL) information.

Future investigations should focus more closely on local interactions between IPPC, dorsal precuneus, and ventral precuneus during mnemonic control. Based on the set of brain regions identified from the functional connectivity analyses, the cingulum bundle emerges as a plausible candidate for a white matter tract that could support retrieval suppression. This tract has been linked previously to long-term memory, in that damage to the cingulum bundle and/or to brain regions connected by it (the retrosplenial and posterior cingulate cortices) can result in amnesia (e.g., Valenstein et al., 1987; von Cramon and Schuri, 1992).

The cingulum bundle stretches from the frontal lobes around the rostrum and genu of the corpus callosum, extending caudally to the parietal lobes and curving ventrally around the splenium, ending in the white matter of posterior parahippocampal gyrus (Schmahmann et al., 2007). Tentatively consistent with the possibility that cortical inputs arrive in posterior MTL via the cingulum bundle, as opposed to the uncinate fasciculus, a tract that connects anterior MTL with orbitofrontal cortex and IPFC, is our observation that task-related modulation was strongest in posterior hippocampus (Fig. 3). The present set of findings led us to hypothesize that the cingulum bundle is involved not only in memory retrieval but also in retrieval suppression; diffusionweighted imaging data are needed to test this novel hypothesis further.

In conclusion, the ability to direct our thought processes (i.e., cognitive control) influences not only what we do but also what we remember. Mnemonic control is mediated by a functional network that links IPFC with the MTL via cingulate and parietal cortices. There are important individual differences in the capacity for mnemonic control that are not accounted for by differences in IQ. Here, we show that tighter coupling within a prefrontal-cingulate-parietal-hippocampal network is associated with more effective intentional suppression of memory retrieval.

\section{References}

Achenbach TM (1991) Manual for the child behavior checklist/4-18 and 1991 profile. Burlington, VT: University of Vermont.

Anderson MC, Green C (2001) Suppressing unwanted memories by executive control. Nature 410:366-369. CrossRef Medline

Anderson MC, Huddleston E (2011) Towards a cognitive and neurobiological model of motivated forgetting. In: True and false recovered memories: toward a reconciliation (Belli RF, ed), Volume 58, pp 53-120. Nebraska symposium on motivation. New York: Springer.

Anderson MC, Ochsner KN, Kuhl B, Cooper J, Robertson E, Gabrieli SW, Glover GH, Gabrieli JD (2004) Neural systems underlying the suppression of unwanted memories. Science 303:232-235. CrossRef Medline

Anderson MC, Reinholz J, Kuhl BA, Mayr U (2011) Intentional suppression of unwanted memories grows more difficult as we age. Psychol Aging 26:397-405. CrossRef Medline

Benoit RG, Anderson MC (2012) Opposing mechanisms support the vol- untary forgetting of unwanted memories. Neuron 76:450-460. CrossRef Medline

Brass M, Derrfuss J, Forstmann B, von Cramon DY (2005) The role of the inferior frontal junction area in cognitive control. Trends Cogn Sci 9:314-316. CrossRef Medline

Bulevich JB, Roediger HL 3rd, Balota DA, Butler AC (2006) Failures to find suppression of episodic memories in the think/no-think paradigm. Mem Cognit 34:1569-1577. CrossRef Medline

Bunge SA, Wright SB (2007) Neurodevelopmental changes in working memory and cognitive control. Curr Opin Neurobiol 17:243-250. CrossRef Medline

Burgund ED, Kang HC, Kelly JE, Buckner RL, Snyder AZ, Petersen SE, Schlaggar BL (2002) The feasibility of a common stereotactic space for children and adults in fMRI studies of development. Neuroimage 17:184200. CrossRef Medline

Butler AJ, James KH (2010) The neural correlates of attempting to suppress negative versus neutral memories. Cogn Affect Behav Neurosci 10:182194. CrossRef Medline

Cabeza R, Ciaramelli E, Olson IR, Moscovitch M (2008) The parietal cortex and episodic memory: an attentional account. Nat Rev Neurosci 9:613625. CrossRef Medline

Cocosco CA, Kollokian V, Kwan RKS, Evans AC (1997) BrainWeb: online interface to a 3D MRI simulated brain database. Neuroimage 5:S425.

Coltheart M (1981) The MRC Psycholinguistic Database. Q J Exp Psychol 33:497-505.

Dale AM (1999) Optimal experimental design for event-related fMRI. Hum Brain Mapp 8:109-114. CrossRef Medline

Davidson MC, Amso D, Anderson LC, Diamond A (2006) Development of cognitive control and executive functions from 4 to 13 years: evidence from manipulations of memory, inhibition, and task switching. Neuropsychologia 44:2037-2078. CrossRef Medline

Depue BE (2012) A neuroanatomical model of prefrontal inhibitory modulation of memory retrieval. Neurosci Biobehav Rev 36:1382-1399. CrossRef Medline

Depue BE, Banich MT, Curran T (2006) Suppression of emotional and nonemotional content in memory: effects of repetition on cognitive control. Psychol Sci 17:441-447. CrossRef Medline

Depue BE, Curran T, Banich MT (2007) Prefrontal regions orchestrate suppression of emotional memories via a two-phase process. Science 37:215219. Medline

Depue BE, Burgess GC, Willcutt EG, Ruzic L, Banich MT (2010) Inhibitory control of memory retrieval and motor processing associated with the right lateral prefrontal cortex: evidence from deficits in individuals with ADHD. Neuropsychologia 48:3909-3917. CrossRef Medline

Detre GJ, Natarajan A, Gershman SJ, Norman K (2013) Moderate levels of activation lead to forgetting in the Think/No-Think paradigm. In press.

Freud S (1966) The standard edition of the complete psychological works of Sigmund Freud. London: Hogarth.

Hanslmayr S, Leipold P, Pastötter B, Bäuml KH (2009) Anticipatory signatures of voluntary memory suppression. J Neurosci 29:2742-2747. CrossRef Medline

Harnishfeger KK, Pope RS (1996) Intending to forget: the development of cognitive inhibition in directed forgetting. J Exp Child Psychol 62:292315. CrossRef Medline

Hutchinson JB, Uncapher MR, Wagner AD (2009) Posterior parietal cortex and episodic retrieval: convergent and divergent effects of attention and memory. Learn Mem 23:343-356. CrossRef

Joormann J, Hertel PT, LeMoult J, Gotlib IH (2009) Training forgetting of negative material in depression. J Abnorm Psychol 118:34-43. CrossRef Medline

Kang HC, Burgund ED, Lugar HM, Petersen SE, Schlaggar BL (2003) Comparison of functional activation foci in children and adults using a common stereotactic space. Neuroimage 19:16-28. CrossRef Medline

Levy BJ, Anderson MC (2008) Individual differences in the suppression of unwanted memories: the executive deficit hypothesis. Acta Psychol 127: 623-635. CrossRef Medline

Levy BJ, Anderson MC (2012) Purging of memories from conscious awareness tracked in the human brain. J Neurosci 32:16785-16794. CrossRef Medline

Luna B, Garver KE, Urban TA, Lazar NA, Sweeney JA (2004) Maturation of cognitive processes from late childhood to adulthood. Child Dev 75: 1357-1372. CrossRef Medline 
Luria AR (1968) The mind of a mnemonist: a little book about a vast memory. Cambridge, MA: Harvard UP.

MacLeod CM (1999) The item and list methods of directed forgetting: test differences and the role of demand characteristics. Psychon Bull Rev 6:123-129. CrossRef Medline

Murray BD, Muscatell KA, Kensinger EA (2011) Effects of emotion and age on performance during a think/no-think memory task. Psychol Aging 26:940-955. CrossRef Medline

Nelson DL, McEvoy CL, Schreiber TA (2004) The University of South Florida free association, rhyme, and word fragment norms. Behav Res Methods Inst Comput 36:402-407. CrossRef Medline

Olson IR, Berryhill M (2009) Some surprising findings on the involvement of the parietal lobe in human memory. Neurobiol Learn Mem 91:155-165. CrossRef Medline

Paz-Alonso PM, Ghetti S, Matlen BJ, Anderson MC, Bunge SA (2009) Memory suppression is an active process that improves over childhood. Front Hum Neurosci 3:24. CrossRef Medline

Rissman J, Gazzaley A, D’Esposito M (2004) Measuring functional connectivity during distinct stages of a cognitive task. Neuroimage 23:752-763. CrossRef Medline

Schmahmann JD, Pandya DN, Wang R, Dai G, D'Arceuil HE, de Crespigny AJ, Wedeen VJ (2007) Association fibre pathways of the brain: parallel observations from diffusion spectrum imaging and autoradiography. Brain 130:630-653. CrossRef Medline

Shimamura AP (2011) Episodic retrieval and the cortical binding of relational activity. Cogn Affect Behav Neurosci 11:277-291. CrossRef Medline

Talairach J, Tourneaux P (1988) Co-planar stereotaxic atlas of the human brain. Stuttgart, Germany: Thieme.

Valenstein E, Bowers D, Verfaellie M, Heilman KM, Day A, Watson RT (1987) Retrosplenial amnesia. Brain 110:1631-1646. CrossRef Medline

von Cramon DY, Schuri U (1992) The septo-hippocampal pathways and their relevance to human memory: a case report. Cortex 28:411-422. Medline

Wegner DM (2009) How to think, say, or do precisely the worst thing for any occasion. Science 325:48-50. CrossRef Medline

Wendelken C, Baym CL, Gazzaley A, Bunge SA (2011) Neural indices of improved attentional modulation over middle childhood. Dev Cogn Neurosci 1:175-186. CrossRef Medline

Wilson SP, Kipp K (1998) The development of efficient inhibition: evidence from directed-forgetting tasks. Dev Rev 18:86-123. CrossRef

Woodcock RW, Mather N, McGrew KS (2001) Woodcock-Johnson III Tests of Cognitive Abilities Examiner's Manual. Itasca, IL: Riverside. 